\title{
Surface decoration by Spirulina polysaccharide enhances the cellular uptake and anticancer efficacy of selenium nanoparticles
}

This article was published in the following Dove Press journal:

International Journal of Nanomedicine

16 February 2012

Number of times this article has been viewed

\author{
Fang Yang ${ }^{1 *}$ \\ Quanming Tang ${ }^{1,2 *}$ \\ Xueyun Zhong ${ }^{3}$ \\ Yan Bai' \\ Tianfeng Chen' \\ Yibo Zhang' \\ Yinghua $\mathrm{Li}^{\prime}$ \\ Wenjie Zheng' \\ 'Department of Chemistry, Jinan \\ University, Guangzhou, China; ${ }^{2}$ South \\ China Seas Institute of Oceanology, \\ Chinese Academy of Sciences, \\ Guangzhou, China; ${ }^{3}$ Department \\ of Pathology, Jinan University, \\ Guangzhou, China \\ *These authors contributed equally \\ to this work
}

Correspondence: Tianfeng Chen and

Wenjie Zheng

No 60I Huangpu Road,

Guangzhou 510632, China

Tel +862085225962

Fax +86 $208522 \quad$ I263

Email felixchentf@gmail.com

Xueyun Zhong

No 60I Huangpu Road, Guangzhou

510632, China

Tel +86 2085220252

Fax +86 $208522 \quad$ I 263

Email tzxy@jnu.edu.cn

\begin{abstract}
A simple and solution-phase method for functionalization of selenium nanoparticles (SeNPs) with Spirulina polysaccharides (SPS) has been developed in the present study. The cellular uptake and anticancer activity of SPS-SeNPs were also evaluated. Monodisperse and homogeneous spherical SPS-SeNPs with diameters ranging from $20 \mathrm{~nm}$ to $50 \mathrm{~nm}$ were achieved under optimized conditions, which were stable in the solution phase for at least 3 months. SPS surface decoration significantly enhanced the cellular uptake and cytotoxicity of SeNPs toward several human cancer cell lines. A375 human melanoma cells were found extremely susceptible to SPS-SeNPs with half maximal (50\%) inhibitory concentration value of $7.94 \mu \mathrm{M}$. Investigation of the underlying mechanisms revealed that SPS-SeNPs inhibited cancer cell growth through induction of apoptosis, as evidenced by an increase in sub- $\mathrm{G}_{1}$ cell population, deoxyribonucleic acid fragmentation, chromatin condensation, and phosphatidylserine translocation. Results suggest that the strategy to use SPS as a surface decorator could be an effective way to enhance the cellular uptake and anticancer efficacy of nanomaterials. SPS-SeNPs may be a potential candidate for further evaluation as a chemopreventive and chemotherapeutic agent against human cancers.
\end{abstract}

Keywords: selenium nanoparticles, Spirulina polysaccharide, cellular uptake, anticancer, apoptosis

\section{Introduction}

A confluence of different types of evidence indicates that the essential nutrient selenium (Se) can affect cancer risk and is a cancer chemopreventive agent. ${ }^{1,2}$ Therefore, cancer chemopreventive and chemotherapeutic effects of Se-containing compounds have been widely studied and have achieved strong support by a majority of epidemiological, preclinical, and clinical studies. ${ }^{3}$ It has been confirmed that the bioavailability and side effects of Se are closely related to its chemical species. ${ }^{4}$ Recently, Se nanoparticles (SeNPs) are receiving additional attention due to their excellent biological activities and low toxicity. ${ }^{5,6}$

Cancer nanotechnology - a crossdisciplinary scientific field merging chemistry, biology, bioengineering, and medicine - provides unique approaches and a comprehensive technology against cancer via molecular imaging, molecular diagnosis, and targeted therapy. ${ }^{7}$ The basic premise is that nanometer-sized particles have unique optical, magnetic, chemical, and structural properties that are not attainable from either individual molecules or bulk solids. ${ }^{8}$ The advancement in multifunctional bionanomaterials makes it possible to diagnose and treat diseases that have traditionally been recognized as incurable via basic therapies or surgical methods, such as malignant cancers. ${ }^{9}$ 
Polysaccharides, a class of biopolymers naturallyoriginated from plants or animals, have been widely studied in biotechnological and biomedical applications. ${ }^{10,11}$ There are three main reasons for the wide application of polysaccharides as functionalizing agents in cancer nanotechnology. Firstly, naturally-originated polysaccharides exhibit unique properties including excellent biocompatibility, biodegradability, stability, and nontoxicity, which are the basic characteristics for polymers used as biomaterials. ${ }^{12}$ Secondly, a number of applications of cancer nanotechnology are based upon the interaction of nanomaterials with the cell and its lipid bilayers. This interaction is mainly controlled by surface properties of the nanoparticles. ${ }^{13}$ The control of the interaction is usually established by means of functionalization of the particles via hybridization of biological molecules and modification of surface properties. ${ }^{14}$ In many cases, the surface of nanoparticles has to be highly hydrophilic and able to prevent protein adsorption. ${ }^{7}$ With hydrophilic groups such as hydroxyl, carboxyl, and amino groups, most natural polysaccharides can attach to biological tissues (mainly epithelia and mucous membranes) via noncovalent bonds. Thirdly, nanoparticles functionalized with bioadhesive polysaccharides could prolong residence time and therefore increase absorption of the loaded drugs. ${ }^{15}$ Although nanoparticles tend to accumulate in cancer cells through passive targeting process, this passive strategy has limitations due to its nonspecific delivery mode. However, when nanoparticles are functionalized with polysaccharides, polysaccharide moieties can interact specifically with the biological targets, eg, lectins that have been found in the surface of tumor cells and malignant tissues. ${ }^{16}$ Therefore, surface decoration of nanomaterials by polysaccharides could enhance cell-permeating and cancer-targeting abilities. ${ }^{7}$

In a previous study, SeNPs were decorated with a monosaccharide sialic acid and the as-prepared nanoparticles showed distinguished cell internalization and selectivity between cancer and normal cells. But the drawback in stability could limit its further applications. ${ }^{17}$ Spirulina polysaccharide (SPS) from food-grade blue-green microalga Spirulina platensis is believed to have many biological functions, such as deoxyribonucleic acid (DNA) repairing effect, immunostimulatory effect, free radical scavenging activity, and antiviral effect. ${ }^{18,19}$ Therefore, SPS could be used as a surface decorator of nanomaterials to prevent plasma protein adsorption, maximize circulation time, and to enhance their cell-penetrating abilities. In the present study, SeNPs functionalized by SPS (SPS-SeNPs) were prepared in an attempt to improve the cancer-targeting, stability, and cellular uptake of SeNPs. The effect of SPS on the anticancer activity of SeNPs against human cancer cells was also evaluated. The change in cell cycle distribution in response to SPS-SeNPs treatments was also examined by flow cytometric analysis to elucidate the underlying mechanisms. Results showed that SPS surface decoration significantly enhanced the cell-penetrating and apoptosis-inducing abilities of SeNPs in A375 human melanoma cells. Taken together, the results suggest that the strategy to use SPS as a surface decorator could be an effective way to enhance the cellular uptake and anticancer efficacy of nanomaterials. SPS-SeNPs may be a potential candidate for further evaluation as a chemopreventive and chemotherapeutic agent against human cancers.

\section{Methods}

\section{Preparation of SPS}

S. platensis was obtained from Research Center of Hydrobiology of Jinan University (Jinan, China) and cultured in Zarrouk medium in the authors' laboratory. ${ }^{20}$ The SPS was prepared as described previously, ${ }^{21}$ with some modifications. Briefly, powdered S. platensis was extracted with hot water at $80^{\circ} \mathrm{C}$ for 4 hours. After concentration and deproteination with Sevag method, the resulting aqueous fraction was dialyzed and precipitated by ethanol. The precipitate was washed with acetone and freeze-dried to yield brown powder, which was then dissolved in water and further purified using diethylaminoethyl-cellulose 52 ion-exchange chromatography, with water as eluent. The partitions containing polysaccharide detected using phenol-sulfuric acid agent were combined, dialyzed, and precipitated with ethanol again. The precipitate was freeze-dried for the second time to yield a white powder, which was SPS with a purity of $98 \%$ determined by using the phenol-sulfuric acid method. ${ }^{22}$

\section{Preparation and characterization of SPS-SeNPs}

Different volumes $(0-1 \mathrm{~mL})$ of SPS stock solution $(1000 \mathrm{mg} / \mathrm{L})$ were mixed with $5 \mathrm{~mL}$ sodium selenite solution $\left(2 \mathrm{mM}\right.$ ) and added to $6 \mathrm{~mL}$ Milli- $\mathrm{Q}^{\mathrm{TM}}$ water (Millipore Corporation, Billerica, MA). Then, $4 \mathrm{~mL}$ fresh ascorbic acid solution $(10 \mathrm{mM})$ was slowly added into the mixtures under magnetic stirring. After reaction for 24 hours, the solutions were dialyzed against Milli-Q water at $4^{\circ} \mathrm{C}$ with water changing every 24 hours until no Se was detected in the outer solutions, as determined by inductively coupled plasma atomic emission spectroscopy (ICP-AES) analysis..$^{20}$ The prepared products were characterized with various spectroscopic and microscopic methods. 
Samples for transmission electron microscopy (TEM) were prepared by dispersing the samples onto a holey carbon film on copper grids. The micrographs were obtained on an H-7650 TEM (Hitachi High Technologies, Tokyo, Japan) operated at an accelerating voltage at $80 \mathrm{kV}$. X-ray diffraction patterns were obtained on an MSAL-XD2 using copper $\mathrm{K} \alpha$, nickel-filter plate radiation at a scan rate of $2 \%$ minute. The Raman spectrum was produced at room temperature on an inVia Raman microscope (Renishaw, Gloucestershire, United Kingdom) equipped with an argon-ion laser at an excitation wavelength of $514.5 \mathrm{~nm}$. Scanning electron microscopy and energy dispersive $\mathrm{X}$-ray analysis was carried out on an EX-250 system (HORIBA Ltd, Kyoto, Japan) and employed to examine the elemental composition of SPSSeNPs. For Fourier transform infrared spectroscopy (FTIR) measurements, the prepared SPS-SeNPs were washed three times using Milli-Q water by centrifugation at $8000 \mathrm{rpm}$ for 20 minutes to remove any free SPS, then the recovered nanoparticles were freeze-dried. Samples for FTIR were conducted by using potassium bromide pellet method and detected by EQUINOX 55 FTIR spectrometer (Bruker Optik $\mathrm{GmbH}$, Ettlingen, Germany). The size distribution of SPSSeNPs was determined using photon correlation spectroscopy with a Zetasizer ${ }^{\circledR}$ Nano ZS instrument (Malvern Instruments Ltd, Malvern, United Kingdom). The results were analyzed by using a built-in software provided by Malvern Instruments. Measurements were carried out in triplicate at a $90^{\circ}$ angle at $25^{\circ} \mathrm{C}$ under suitable dilution conditions. The size distribution of SPS-SeNPs prepared under $50 \mathrm{mg} / \mathrm{L}$ SPS was determined at different time intervals.

\section{Cell culture and cell viability assay}

Several human cancer cell lines, including A375 melanoma cells, human cervical carcinoma HeLa-229 cells, MCF-7 breast adenocarcinoma cells, osteosarcoma MG-63, and human normal kidney HK-2 cells were all purchased from American Type Culture Collection (Manassas, VA). All cells were maintained in Dulbecco's modified Eagle medium supplemented with fetal bovine serum (10\%), penicillin, and streptomycin at $37^{\circ} \mathrm{C}$ in a humidified incubator with $5 \%$ carbon dioxide atmosphere.

Cell viability was determined by measuring the ability of cells to transform thiazolyl blue tetrazolium bromide (methylthiazol tetrazolium) to a purple formazan dye as described. ${ }^{17}$ Cells were seeded in 96-well tissue culture plates at $2.5 \times 10^{3}$ cells/well for 24 hours. The cells were then incubated with SPS-SeNPs at different concentrations for different periods of time. After incubation, $20 \mu \mathrm{L} /$ well of methylthiazol tetrazolium solution $(5 \mathrm{mg} / \mathrm{mL}$ phosphate buffered saline [PBS]) was added and incubated for an additional 5 hours. The medium was aspirated and replaced with $150 \mu \mathrm{L} /$ well dimethyl sulfoxide to dissolve the salt formed formazan. The color intensity of the formazan solution, which reflects the cell growth condition, was measured at $570 \mathrm{~nm}$ using a microplate spectrophotometer (SpectraMax ${ }^{\circledR} 250$; Molecular Devices Inc, Sunnyvale, CA).

\section{Determination of Se intracellular uptake}

A375 cells were harvested by centrifugation after incubation at different concentrations of SPS-SeNPs for 72 hours, and then washed with PBS buffer three times to remove any extracellular Se. The intracellular Se concentration was determined by ICP-AES method, as previously described. ${ }^{20}$ Briefly, collected cells were digested with $3 \mathrm{~mL}$ concentrated nitric acid and $1 \mathrm{~mL}$ hydrogen peroxide in an infrared rapid digestion system (Gerhardt, Herlev, Denmark) at $180^{\circ} \mathrm{C}$ for 1.5 hours. The digested solution was reconstituted to $10 \mathrm{~mL}$ with Milli-Q water prior to ICP-AES analysis.

\section{Flow cytometric analysis}

Cell cycle distribution was analyzed by flow cytometry, as previously described. ${ }^{23}$ After treatment with SPS-SeNPs for 24 hours, the cells were trypsinized, washed with PBS buffer ( $\mathrm{pH} 7.4$ ), and fixed with $70 \%$ ethanol overnight at $-20^{\circ} \mathrm{C}$. The fixed cells were washed with the same buffer and stained with propidium iodide working solution $(1.21 \mathrm{mg} / \mathrm{mL}$ tris-hydroxymethyl aminomethane, $700 \mathrm{U} / \mathrm{mL}$ ribonuclease, $50.1 \mu \mathrm{g} / \mathrm{mL}$ propidium iodide; $\mathrm{pH} 8.0$ ) for 4 hours in darkness. The stained cells were analyzed on a flow cytometer (Beckman Coulter Inc, Brea, CA). Cell cycle distribution was examined using MultiCycle software (Phoenix Flow Systems, San Diego, CA). The proportion of cells in $G_{0} / G_{1}, S$, and $G_{2} / M$ phases was represented as DNA histograms. Apoptotic cells with hypodiploid DNA content were measured by quantifying the sub- $\mathrm{G}_{1}$ peak in the cell cycle pattern. For each experiment, 10,000 events per sample were recorded.

\section{Annexin-V-FLUOS and 4',6-diamidino-2- phenylindole (DAPI) staining assay}

Translocation of phosphatidylserine in plasma membrane of cells treated with SPS-SeNPs was determined using AnnexinV-FLUOS staining assay (Roche Applied Science, Penzberg, Germany). The cells were seeded in eight-chamber polystyrene vessel (Becton Dickinson and Company, Franklin Lakes, NJ) and incubated for 24 hours. After addition of 
SPS-SeNPs to the culture medium, incubation continued for an additional 24 hours. Cells were then stained with Annexin-V-FLUOS labeling solution (Annexin-V-fluorescein in 4-[2-hydroxyethyl]-1-piperazineethanesulfonic acid buffer containing propidium iodide) for 15 minutes. The slides were directly analyzed by confocal microscopy (Radiance 2100MP scanning system equipped with $488 \mathrm{~nm}$ argon laser; Bio-Rad Laboratories, Hercules, CA) and fluorescence images were captured using LaserShape 2000 software. Excitation wavelength was set at $488 \mathrm{~nm}$, while emission wavelengths were set at $515 \mathrm{~nm}$ and $617 \mathrm{~nm}$ for Annexin-V-fluorescein and propidium iodide, respectively. For DAPI staining assay, cells cultured in chamber slides were fixed with $3.7 \%$ formaldehyde for 10 minutes and permeabilized with $0.1 \%$ Triton $\mathrm{X}-100$ in PBS. And then, the nuclei were stained with DAPI for 15 minutes at $37^{\circ} \mathrm{C}$. After washing with PBS, the cells were observed under fluorescence microscope (Eclipse 80i; Nikon Corporation, Tokyo, Japan).

\section{Statistical analysis}

Data were expressed as mean \pm standard deviation from at least three independent experiments. Difference between two groups was analyzed by two-tailed Student's $t$-test and the significance among three or more groups was analyzed by one-way analysis of variance multiple comparisons. In all analyses, a value of $P<0.05$ was considered statistically significant.

\section{Results and discussion Preparation and characterization of SPS-SeNPs}

In the present study, SPS-SeNPs were prepared in a simple, safe, and solution-phase redox system, in which sodium selenite was firstly well distributed in SPS aqueous solution. Then the reductant ascorbic acid solution was added to initiate the reaction with sodium selenite. The mixture turned red gradually, indicating that the elemental SeNPs had been prepared. Because the size of the nanoparticles is an essential issue for its medical application and the concentrations of the capping agents play an important role in the controlling of the size of nanoparticles, ${ }^{24}$ the effects of SPS concentrations on the morphology and size distribution of SeNPs were investigated. Figure 1 shows the TEM images of the samples prepared under different SPS concentrations at the reaction time of 24 hours. It can be observed that the morphology of the products was affected significantly by SPS concentrations. In the absence of SPS, the prepared SeNPs aggregated easily and their size was not uniform, ranging from $90 \mathrm{~nm}$ to $550 \mathrm{~nm}$ (Figure 1A). With the increase in SPS concentrations from $1 \mathrm{mg} / \mathrm{L}$ to $10 \mathrm{mg} / \mathrm{L}$
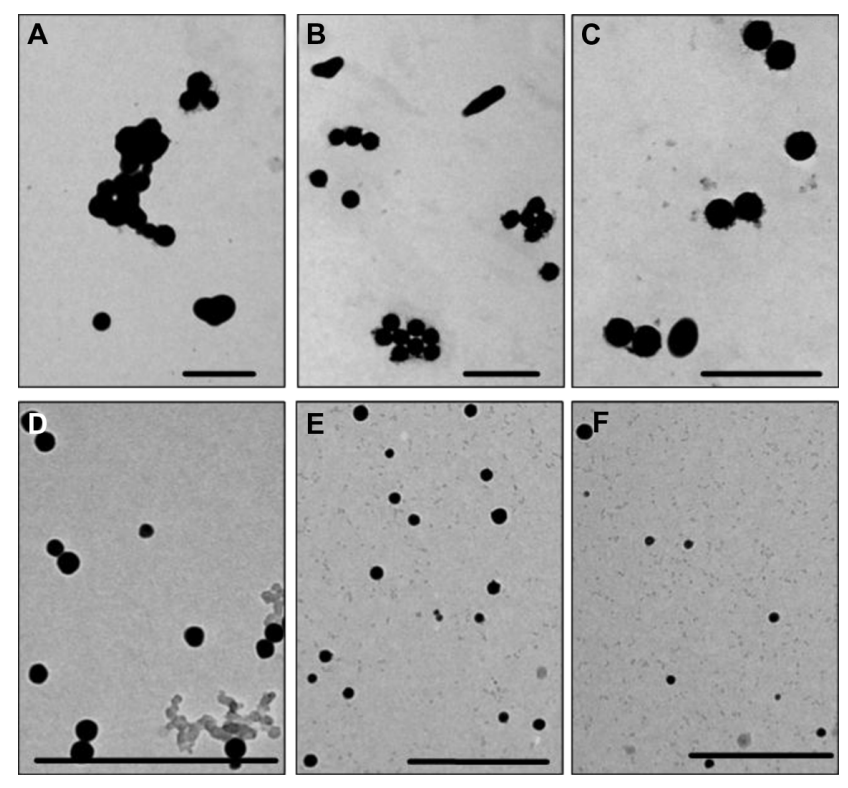

Figure I Transmission electron microscopic images of selenium nanoparticles obtained at different Spirulina polysaccharide concentrations at the reaction time of 24 hours: (A) $0 \mathrm{mg} / \mathrm{L}$; (B) $1 \mathrm{mg} / \mathrm{L} ;$ (C) $5 \mathrm{mg} / \mathrm{L}$; (D) $10 \mathrm{mg} / \mathrm{L}$; (E) $50 \mathrm{mg} / \mathrm{L}$; (F) $100 \mathrm{mg} / \mathrm{L}$.

Note: Scale bar $500 \mathrm{~nm}$.

(Figure 1B-D), uniformity of prepared SPS-SeNPs gradually improved and the average diameter of SPS-SeNPs decreased. When SPS concentration reached $50 \mathrm{mg} / \mathrm{L}$, SPS-SeNPs presented a homogeneous spherical structure with a diameter of about 20-50 nm (Figure 1E). A further increase in SPS concentration to $100 \mathrm{mg} / \mathrm{L}$ did not affect the diameter, but resulted in the reduction in yield of production of SeNPs (Figure 1F). Therefore, SPS-SeNPs prepared under $50 \mathrm{mg} / \mathrm{L}$ of SPS were used for the following experiments.

$\mathrm{X}$-ray diffraction analysis was performed to examine the phase of the prepared SPS-SeNPs. Figure 2A shows a typical X-ray diffraction pattern of SPS-SeNPs, which clearly reveals that the polymorph of the obtained SeNPs is amorphous Se. ${ }^{25}$ Figure 2B demonstrates the Raman spectrum of SPS-SeNPs. The resonance peak at $253 \mathrm{~cm}^{-1}$ was a characteristic absorption band for monoclinic Se and amorphous Se. ${ }^{26}$ Furthermore, scanning electron microscopy and energy dispersive X-ray was employed to analyze the elemental chemical composition of SPS-SeNPs (Figure 2C). Three signals could be observed from the energy dispersive X-ray diagram: a strong signal from the Se atom $(86.30 \%)$ together with the SPS signals of $\mathrm{O}$ atom $(3.25 \%)$ and $\mathrm{C}$ atom (10.45\%). There were no obvious peaks for other elements or impurities.

FTIR analysis was also used to characterize the interaction between SeNPs and SPS in the as-prepared nanomaterials. Figure $3 \mathrm{~A}$ and $\mathrm{B}$ corresponded to the spectra of SPS and SPS-SeNPs, respectively. The characteristic stretching 
A

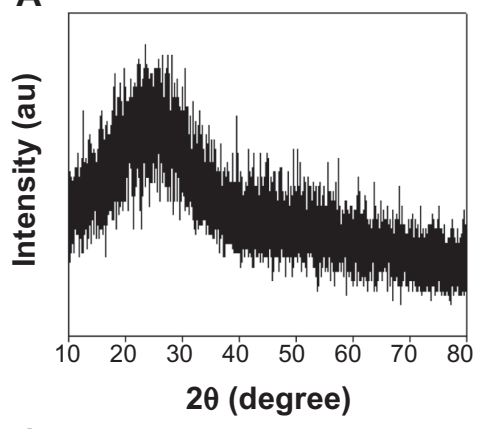

B

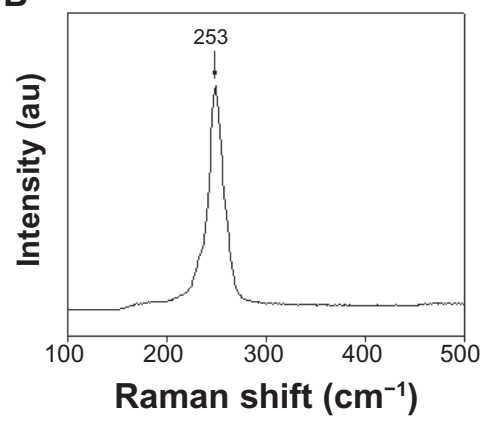

C

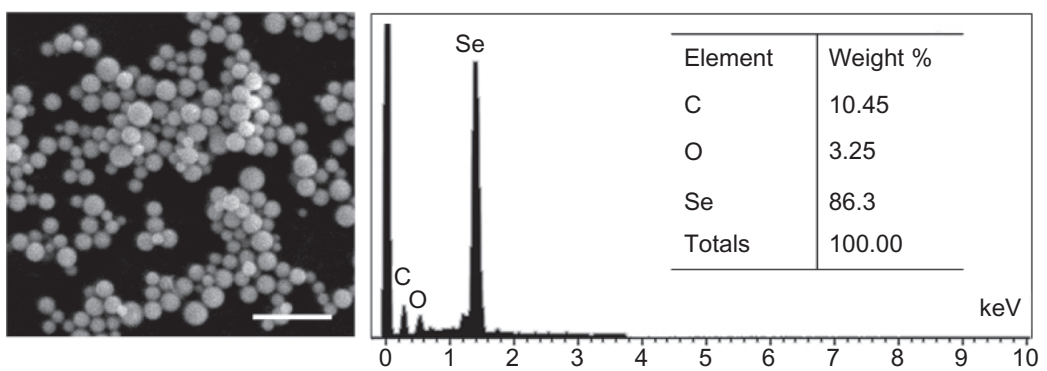

Figure 2 (A) X-ray diffraction pattern, (B) Raman spectrum, and (C) scanning electron microscopy and energy dispersive X-ray analysis of selenium nanoparticles functionalized by Spirulina polysaccharide.

Abbreviations: au, absorbance units; C, carbon; O, oxygen; Se, selenium.

vibrations of the hydroxyl group $(-\mathrm{OH})$ were observed in the two spectra, but the absorption bands of $-\mathrm{OH}$ at $3446 \mathrm{~nm}$ in Figure 3B shifted slightly to $3438 \mathrm{~nm}$ (Figure 3A). These results suggest that some weak interaction existed between SeNPs and SPS. The weakening of absorption intensity of - $\mathrm{OH}$ in Figure 3B was also observed as compared with that in Figure 3A, indicating that free-OH decreased when SPS was linked with Se. ${ }^{26}$ The appearance of absorption bands in

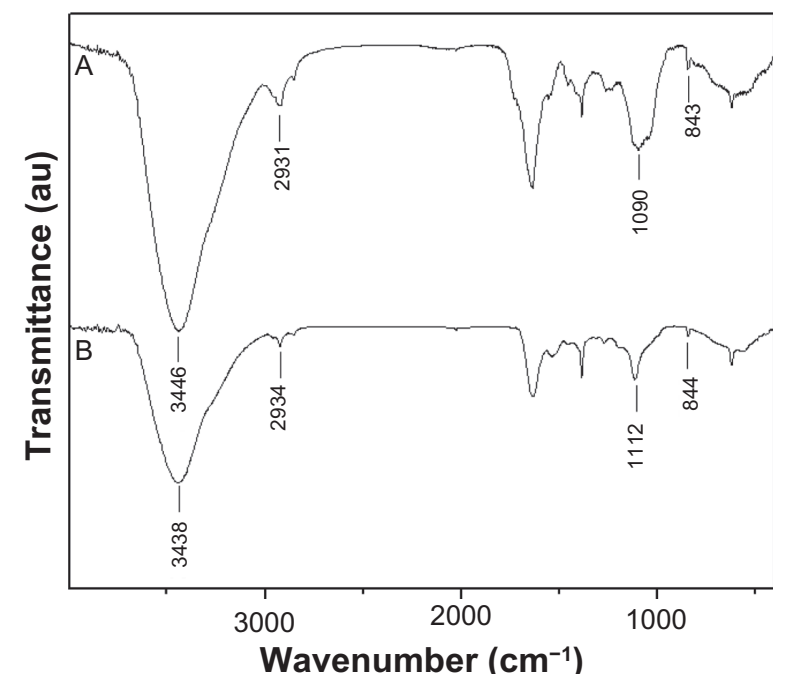

Figure 3 Fourier transform infrared spectra of (A) Spirulina polysaccharide and (B) selenium nanoparticles functionalized by Spirulina polysaccharide. Abbreviation: au, absorbance units.
Figure 3B at $2934 \mathrm{~nm}, 1112 \mathrm{~nm}$, and $844 \mathrm{~nm}$, which were assigned to stretching vibration of aliphatic $\mathrm{C}-\mathrm{H}, \mathrm{C}-\mathrm{O}-\mathrm{H}$, and $\alpha$-D-glucose in polysaccharide, respectively, further confirmed the conjunction of SPS with SeNPs. ${ }^{27}$

The stability of nanoparticles was another important issue for its future medical applications. ${ }^{28}$ Figure $4 \mathrm{~A}$ showed average size of SPS-SeNPs obtained at different SPS concentrations. The average particle size at reaction time of 24 hours was 535, 157, 99.1, 88.4, 48.6, and $45.7 \mathrm{~nm}$ when SPS concentrations were $0,1,5,10,50$, and $100 \mathrm{mg} / \mathrm{L}$, respectively. These results indicate that the optimum SPS concentration for preparation of homogeneous spherical SPS-SeNPs was $50 \mathrm{mg} / \mathrm{L}$, which was in accordance with the results of TEM observation. The stability of SPS-SeNPs prepared at $50 \mathrm{mg} / \mathrm{L}$ SPS concentration (Figure 4B) was further investigated, and the results show that SPS-SeNPs could remain stable in the solution phase for at least 3 months.

Based on the above results, it was concluded that highpurity and stable SPS-SeNPs were obtained, and a functionalization mechanism was proposed as illustrated in Figure 5. To prepare the product SPS-SeNPs, SPS, sodium selenite, and ascorbic acid were added sequentially and the following reaction would occur. Firstly, selenite would form hydrous ions in water, and the $-\mathrm{OH}$ from water on the surface of the hydrous ions could form hydrogen bonds with the $-\mathrm{OH}$ from SPS, resulting in the distribution of selenite along the flexible 
A

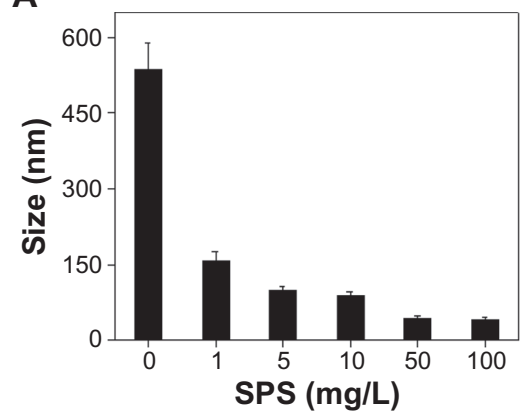

B

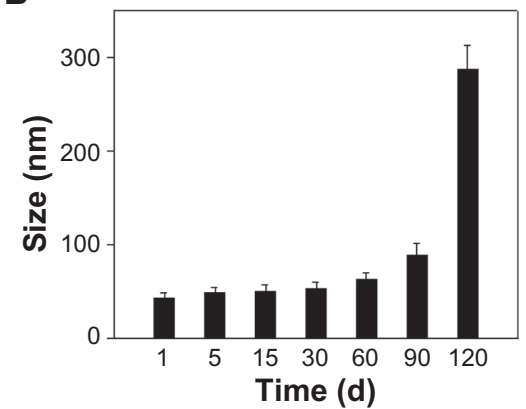

Figure 4 (A) Size distribution of selenium nanoparticles obtained at different Spirulina polysaccharide concentrations at the reaction time of 24 hours and (B) time-course of size distribution of selenium nanoparticles functionalized by Spirulina polysaccharide obtained at $50 \mathrm{mg} / \mathrm{L}$ of Spirulina polysaccharide.

Note: Each value represents mean \pm standard deviation $(n=3)$.

Abbreviations: d, days; SPS, Spirulina polysaccharide.

long chain of polymeric SPS molecules. ${ }^{29}$ Secondly, selenite could be reduced by ascorbic acid to elemental Se. Thirdly, with the amount of elemental Se increasing, Se would agglomerate with each other to form an Se nucleus. Lastly, the Se nucleus would grow into SeNPs by absorbing more Se on its surface so as to reduce the free energy according to the wellknown Gibbs-Thomson law. ${ }^{30}$ Because of the detection of $\mathrm{C}$ and $\mathrm{O}$ signals in scanning electron microscopy and energy dispersive X-ray analysis (Figure 2C) and similarity of FTIR spectra between SPS and SPS-SeNPs (Figure 3), it was proposed that the functionalization of SeNPs with SPS might be a simple physical adsorption due to the large specific surface area of SeNPs. The electronic attraction might also take partial effect in the functionalization process in that the elemental Se contained empty $4 p$ orbital and the oxygen of $-\mathrm{OH}$ in SPS had unpaired electrons. Like other polysaccharides, the characteristics of emulsification and suspension of SPS facilitated the stability of SPS-SeNPs in solution..$^{27,31}$

\section{Cellular uptake of SPS-SeNPs by A375 human melanoma cells}

Like other chemotherapeutics, the effective cytotoxicity of nanomaterial-based therapies usually requires a fairly high level of accumulation within the cancer cells. ${ }^{32}$ Although nanomaterials tend to accumulate in cancer cells through a passive targeting process and often serve as "nanocarriers" for chemotherapeutics, this passive strategy has limitations due to its random delivery mode. ${ }^{7}$ Studies have shown that enhancement of tumor accumulation of nanoparticles can be achieved by increasing the cellular uptake of functionalized nanoparticles. ${ }^{17}$ In recent years many efforts have been made to develop tumor-targeting nanomaterials in an attempt to enhance cellular uptake via functionalization of nanoparticles. The beneficial effects of polysaccharide functionalization on tumor-targeting have been reported previously. ${ }^{33}$ In this work, a technique was proposed to overcome the passive transport drawbacks of nanomaterials by functionalized SeNPs with a biological molecule SPS to enhance the cancer-targeting and cell-penetrating abilities. To examine the cellular uptake of SPS-SeNPs, ICP-AES method was used to determine the Se concentrations in A375 cells after a 72-hour exposure (Figure 6). It was found that treatment with SPS-SeNPs significantly increased intracellular Se concentrations from $6.16 \mu \mathrm{g} / 10^{9}$ (control) to $298.63 \mu \mathrm{g} / 10^{9}$ cells (treated with $20 \mu \mathrm{M}$ SPS-SeNPs), which was 8.98 times higher than the group of SeNPs without SPS decoration $\left(33.24 \mu \mathrm{g} / 10^{9}\right.$ cells $)$.

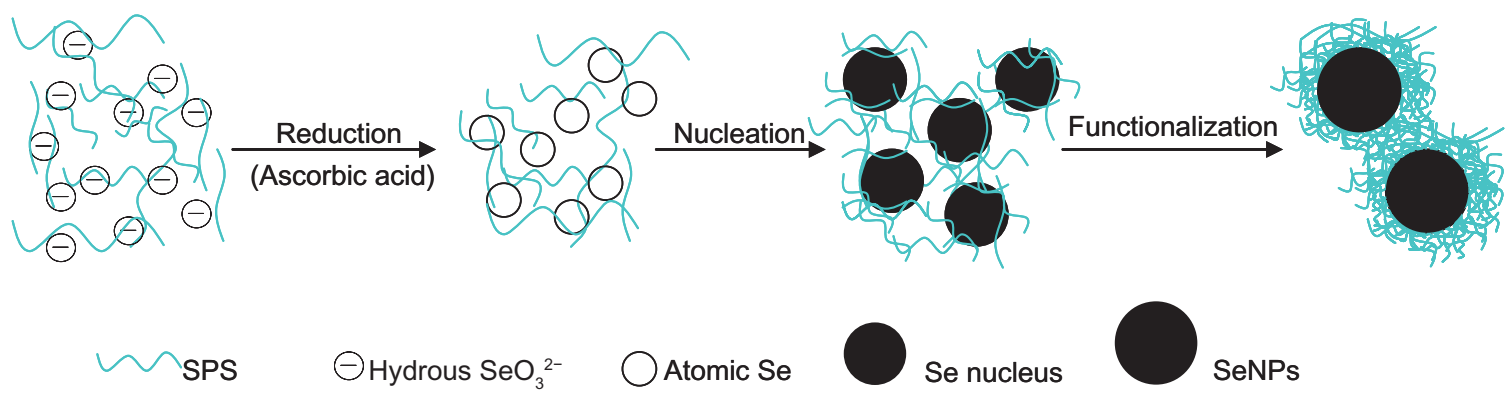

Figure 5 Functionalization of selenium nanoparticles with Spirulina polysaccharide.

Abbreviations: Se, selenium; $\mathrm{SeO}_{3}{ }^{2-}$, selenite; SeNPs, selenium nanoparticles; SPS, Spirulina polysaccharide. 


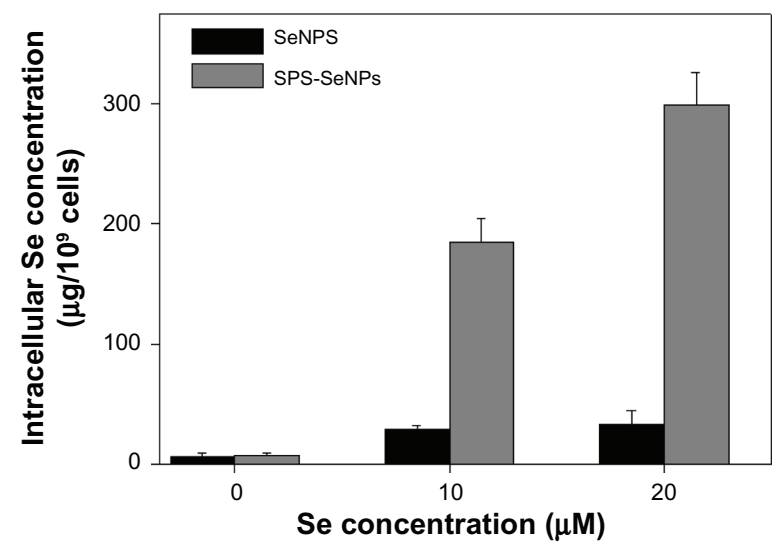

Figure 6 Cellular uptake of selenium nanoparticles functionalized by Spirulina polysaccharide by A375 cells. Cells were exposed to selenium nanoparticles functionalized by Spirulina polysaccharide or selenium nanoparticles at different concentrations for 72 hours. Cells without treatment were used as control. Intracellular selenium concentrations were determined with inductively coupled plasma atomic emission spectroscopy method.

Note: Each value represents mean \pm standard deviation $(n=3)$.

Abbreviations: Se, selenium; SeNPs, selenium nanoparticles; SPS-SeNPs, selenium nanoparticles functionalized by Spirulina polysaccharide.

Moreover, the increase of SPS-SeNPs concentration from $10 \mu \mathrm{M}$ to $20 \mu \mathrm{M}$ caused the corresponding enhancement of intracellular Se concentrations. The results indicate that the functionalization of SPS played an essential role in the uptake of SeNPs in cancer cells in vitro.

\section{In vitro anticancer activity of SPS-SeNPs}

During applications of cancer nanotechnology, active targeting of nanomaterials is usually achieved by conjugation of a targeting component to the surface that provides preferential accumulation of nanomaterials in the tumor-bearing organ, in the tumor itself, individual cancer cells, or intracellular organelles inside cancer cells. This approach is based on specific interactions, such as lectin-carbohydrate, ligandreceptor, and antibody-antigen. Lectin-carbohydrate is one of the classic examples of targeted drug delivery. In the past decade, many studies have demonstrated that the surface of tumor cells and malignant tissues contain lectins. ${ }^{16}$ Therefore, carbohydrate moieties can be used to target drug delivery systems to lectins that exist in the cancer cell surface.

In the present study, SPS was chosen as a carbohydrate moiety to enhance the targeting effects and cell-penetrating activity of SeNPs. As shown in Figure 7, cellular uptake of SPS-SeNPs resulted in a dose-dependent growth inhibition as examined by methylthiazol tetrazolium assay. SPS-SeNPs exhibited a broad spectrum of inhibition against several cancer cell lines, including A375, MCF-7, HeLa-229, and MG-63 (Figure 7A). In contrast, as a negative control, SeNPs without SPS functionalization demonstrated much lower cytotoxic effect on all of the above cancer cells, with half maximal (50\%)

\section{A}
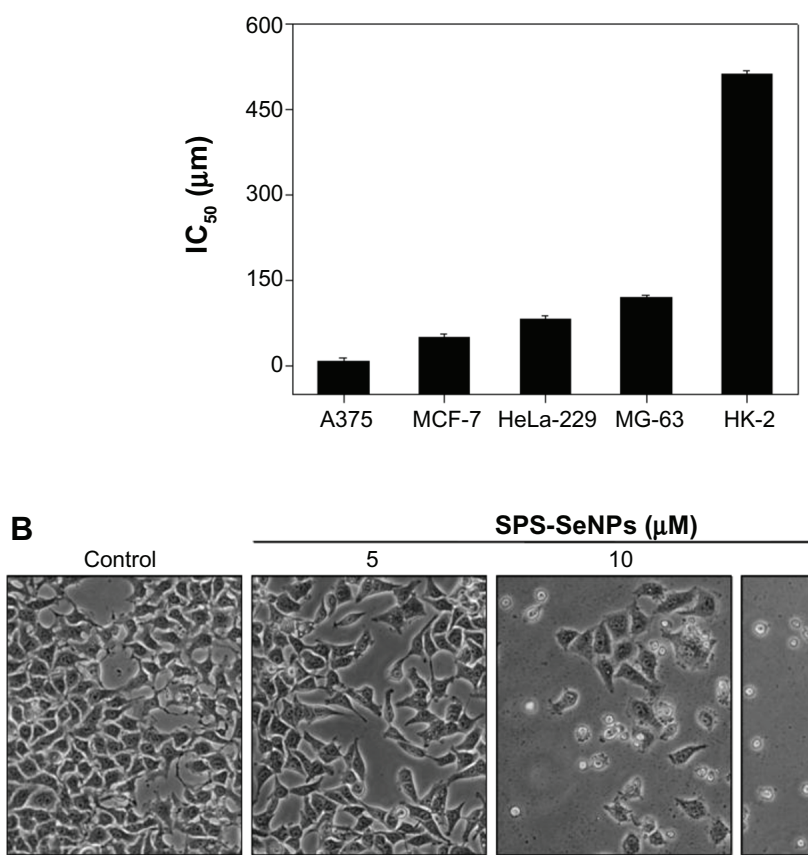

SPS-SeNPs $(\mu \mathrm{M})$
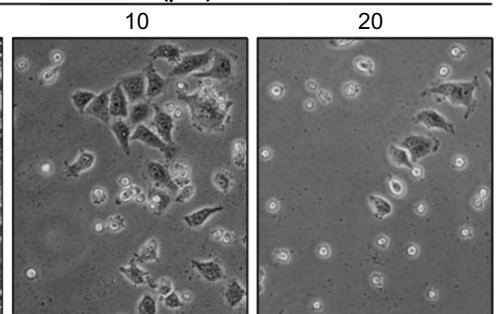

Figure 7 (A) Half maximal inhibitory concentration value of various cancer and normal cell lines after incubation for 72 hours with selenium nanoparticles functionalized by Spirulina polysaccharide and (B) morphological changes in A375 cells observed by phase contrast microscopy (magnification I00X).

Notes: Each value represents mean \pm standard deviation $(n=3) . I C_{50}$ values were based on selenium concentration as determined by inductively coupled plasma atomic emission spectroscopy method.

Abbreviations: $I_{50}$, half maximal (50\%) inhibitory concentration; SPS-SeNPs, selenium nanoparticles functionalized by Spirulina polysaccharide. 
inhibitory concentration against A375 cells at $243.9 \mu \mathrm{M}$. SPS-SeNPs exhibited the highest cytotoxicity against A375 melanoma cells with 50\% inhibitory concentration value at $7.94 \mu \mathrm{M}$, but showed the lowest cytotoxicity toward human normal kidney HK-2 cells. Therefore, the cellular morphological changes of A375 cells upon exposure to SPSSeNPs for 72 hours were also monitored by phase contrast microscopy (Figure 7B). As illustrated in this figure, cells displayed dose-dependent reduction in cell numbers, loss of intercellular contact, cell shrinkage, and formation of apoptotic bodies. In contrast, control cells not treated with SPS-SeNPs remained intact with regularity in shape. These results suggest that SPS-SeNPs possess great selectivity between cancer and normal cells and display potential application in Se supplementation, cancer chemoprevention, and chemotherapy, especially for human melanoma.

\section{SPS-SeNPs induce apoptosis in A375 human melanoma cells}

Several mechanisms have been postulated to elucidate the anticancer action of Se, including induction of apoptosis, effects on the cell cycle distribution, inhibition of angiogenesis, protection against oxidative stress, detoxification of carcinogens, stimulation of the immune system, modulation of thioredoxin reductase activity, and maintenance of cell redox balance. ${ }^{34}$ Among these potential mechanisms of Se action, cell apoptosis receives the most attention and has been postulated to be critical for cancer chemoprevention by selenocompounds. Se could induce extrinsic or intrinsic apoptotic signaling in a number of cancer cell types via alteration of expression or activities of mitochondria-associated proteins, cell cycle regulatory proteins, proteases, signaling proteins, angiogenic factors, and transcription factors. ${ }^{35}$ The main reason for the proliferation inhibition on cancer cells caused by cancer suppressors is the induction of apoptosis or cell cycle arrest, or both of the two biochemical pathways simultaneously. ${ }^{36}$ In order to understand whether apoptosis was involved in cell death induced by SPS-SeNPs, a quantitative in vitro apoptotic detection assay (flow cytometric analysis) was used. The representative DNA histograms (Figure 8A and B), obtained after propidium iodide staining of permeabilized A375 cells exposed to different concentrations of SPS-SeNPs for 24 hours, showed a significant dose-dependent increase in the sub- $\mathrm{G}_{1}$ cells population from $2.4 \%$ (control) to $65.9 \%$ (20 $\mu$ M SPS-SeNPs). No significant changes were observed in the proportion of cells in $\mathrm{G}_{0} / \mathrm{G}_{1}, \mathrm{~S}$, and $\mathrm{G}_{2} / \mathrm{M}$ phases. These results indicate that cell death induced by SPS-SeNPs mainly resulted from cell apoptosis.
The characteristic biochemical hallmarks of apoptosis are phosphatidylserine translocation and nuclear condensation. ${ }^{37}$ Therefore, Annexin-V-FLUOS labeling assay and DAPI staining assay were used to determine the changes of fluorescent intensity for the cells treated without (control) or with SPS-SeNPs (Figure 8C). It was observed that the control cells were Annexin-V-FLUOS-negative and the percentage of Annexin-V-FLUOS-positive cells increased with increasing concentrations of SPS-SeNPs (Figure 8C, upper panel), suggesting that the cells treated with SPS-SeNPs underwent early apoptotic events. The bright clumps
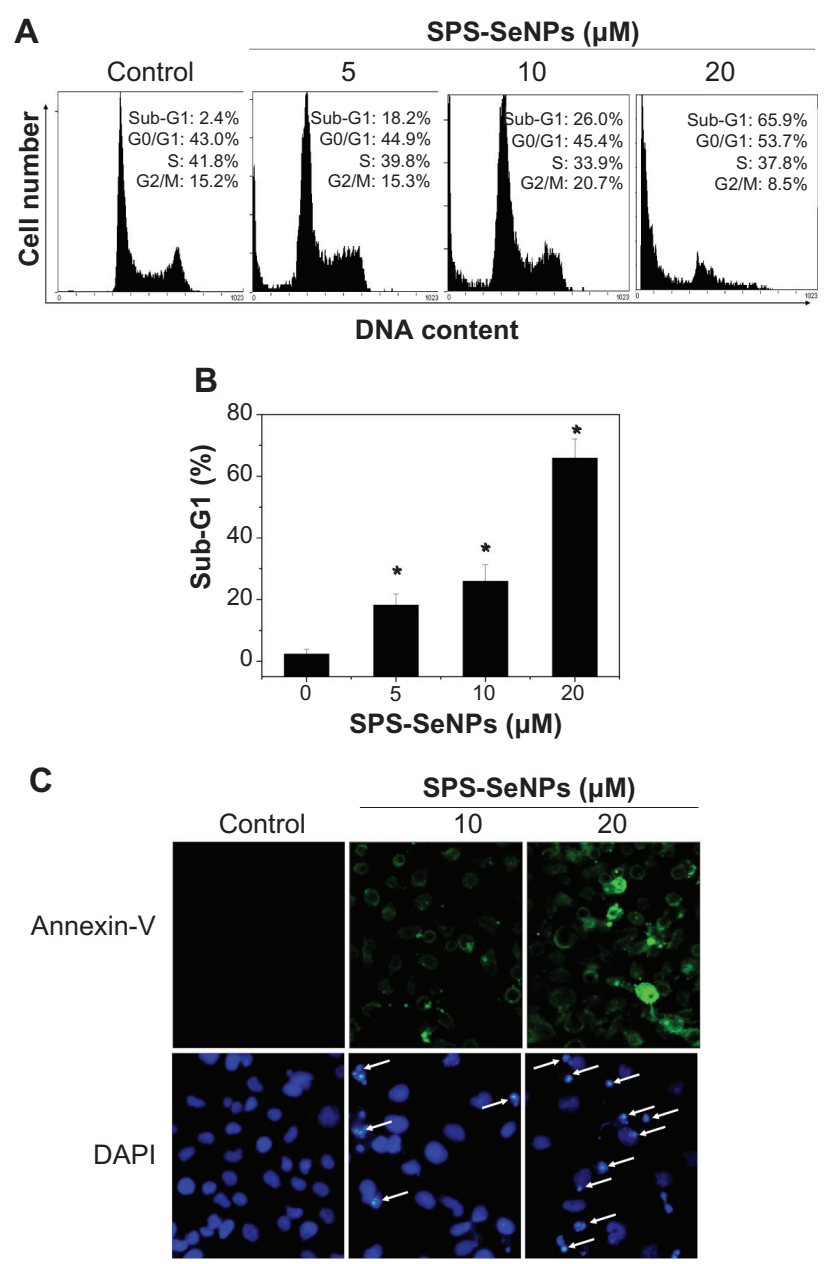

Figure 8 Induction of apoptosis by selenium nanoparticles functionalized by Spirulina polysaccharide. (A) Flow cytometric analysis of cell cycle distribution of A375 cells treated for 24 hours. (B) Quantitative analysis of selenium nanoparticles functionalized by Spirulina polysaccharide induced apoptotic cell death by measuring the sub-G, cell population. Significant difference between selenium nanoparticles functionalized by Spirulina polysaccharide treatment and control is indicated at $P<0.05$ level. (C) Representative images of phosphatidylserine translocation (upper panel) and nuclear condensation (lower panel) in A375 cells exposed to selenium nanoparticles functionalized by Spirulina polysaccharide for 24 hours as examined by Annexin-V-FLUOS labeling assay (magnification 600x) and 4',6-diamidino-2phenylindole staining assay (magnification 600×), respectively.

Note: $* P<0.05$.

Abbreviations: DAPI, 4',6-diamidino-2-phenylindole; DNA, deoxyribonucleic acid; SPS-SeNPs, selenium nanoparticles functionalized by Spirulina polysaccharide. 
(pointed with arrows) of DAPI-positive chromatin in the cells treated with SPS-SeNPs also indicate the process of apoptosis (Figure 8C, lower panel). All of the above results suggest that apoptosis was the main reason for the death of cells treated with SPS-SeNPs. The intracellular molecular mechanisms of cell apoptosis induced by SeNPs may involve a series of biological processes, such as oxidative stress, mitochondrial dysfunction, and activation of caspase $3,{ }^{17,23}$ which need further study.

\section{Conclusion}

In this study, a simple and solution-phase method for functionalization of SeNPs with SPS was demonstrated and their cellular uptake and anticancer activity evaluated. The monodisperse and highly stable SPS-SeNPs were prepared under optimized conditions. SPS surface decoration significantly enhanced the cellular uptake and anticancer efficacy of SeNPs. Induction of apoptosis was found as the major mode of cell death induced by SPS-SeNPs in cancer cells. The results suggest that the strategy to use SPS as a surface decorator could be an effective way to enhance the cellular uptake and anticancer efficacy of nanomaterials. SPS-SeNPs may be a potential candidate for further evaluation as a chemopreventive and chemotherapeutic agent against human cancers.

\section{Acknowledgments}

This work was supported by Natural Science Foundation of China and Guangdong Province, Key Project of Science and Technology Department of Guangdong Province, China Postdoctoral Science Foundation, and the Fundamental Research Funds for the Central Universities.

\section{Disclosure}

The authors report no conflicts of interest in this work.

\section{References}

1. Rayman MP. The importance of selenium to human health. Lancet. 2000;356(9225):233-241.

2. Tran PA, Sarin L, Hurt RH, Webster TJ. Differential effects of nanoselenium doping on healthy and cancerous osteoblasts in coculture on titanium. Int J Nanomedicine. 2010;5:351-358.

3. Sinha R, El-Bayoumy K. Apoptosis is a critical cellular event in cancer chemoprevention and chemotherapy by selenium compounds. Curr Cancer Drug Targets. 2004;4(1):13-28.

4. Ip C, Hayes C, Budnick RM, Ganther HE. Chemical form of selenium, critical metabolites, and cancer prevention. Cancer Res. 1991;51(2):595-600.

5. Wang H, Zhang J, Yu H. Elemental selenium at nano size possesses lower toxicity without compromising the fundamental effect on selenoenzymes: comparison with selenomethionine in mice. Free Radic Biol Med. 2007;42(10):1524-1533.
6. Zhang J, Wang X, Xu T. Elemental selenium at nano size (Nano-Se) as a potential chemopreventive agent with reduced risk of selenium toxicity: comparison with se-methylselenocysteine in mice. Toxicol Sci. 2008;101(1):22-31.

7. Nie S, Xing Y, Kim GJ, Simons JW. Nanotechnology applications in cancer. Annu Rev Biomed Eng. 2007;9:257-288.

8. Nel A, Xia T, Madler L, Li N. Toxic potential of materials at the nanolevel. Science. 2006;311(5761):622-627.

9. Rozhkova EA, Ulasov I, Lai B, Dimitrijevic NM, Lesniak MS, Rajh T. A high-performance nanobio photocatalyst for targeted brain cancer therapy. Nano Lett. 2009;9(9):3337-3342.

10. Kumari A, Yadav SK, Yadav SC. Biodegradable polymeric nanoparticles based drug delivery systems. Colloids Surf B Biointerfaces. 2010;75(1): $1-18$.

11. Zhang SY, Zhang J, Wang HY, Chen HY. Synthesis of selenium nanoparticles in the presence of polysaccharides. Mater Lett. 2004;58(21):2590-2594.

12. Lemarchand C, Gref R, Couvreur P. Polysaccharide-decorated nanoparticles. Eur J Pharm Biopharm. 2004;58(2):327-341.

13. Verma A, Stellacci F. Effect of surface properties on nanoparticle-cell interactions. Small. 2010;6(1):12-21.

14. Sajja HK, East MP, Mao H, Wang YA, Nie S, Yang L. Development of multifunctional nanoparticles for targeted drug delivery and noninvasive imaging of therapeutic effect. Curr Drug Discov Technol. 2009;6(1): $43-51$.

15. Liu Z, Jiao Y, Wang Y, Zhou C, Zhang Z. Polysaccharides-based nanoparticles as drug delivery systems. Adv Drug Deliv Rev. 2008;60(15): $1650-1662$.

16. Lotan R, Raz A. Lectins in cancer cells. Ann N Y Acad Sci. 1988;551: 385-398.

17. Zheng JS, Zheng SY, Zhang YB, et al. Sialic acid surface decoration enhances cellular uptake and apoptosis-inducing activity of selenium nanoparticles. Colloids Surf B Biointerfaces. 2011;83(1):183-187.

18. Pugh N, Ross SA, ElSohly HN, ElSohly MA, Pasco DS. Isolation of three high molecular weight polysaccharide preparations with potent immunostimulatory activity from Spirulina platensis, aphanizomenon flos-aquae and Chlorella pyrenoidosa. Planta Med. 2001;67(8):737-742.

19. Hayashi O, Katoh T, Okuwaki Y. Enhancement of antibody production in mice by dietary Spirulina platensis. J Nutr Sci Vitaminol (Tokyo). 1994;40(5):431-441.

20. Chen T, Wong YS, Zheng W. Purification and characterization of selenium-containing phycocyanin from selenium-enriched Spirulina platensis. Phytochemistry. 2006;67(22):2424-2430.

21. Lee JB, Hayashi T, Hayashi K, et al. Further purification and structural analysis of calcium spirulan from Spirulina platensis. J Nat Prod. 1998;61(9):1101-1104.

22. Dubois M, Gilles KA, Hamilton JK, Rebers PA, Smith F. Colorimetric method for determination of sugars and related substances. Anal Chem. 1956;28(3):350-356.

23. Chen T, Wong YS, Zheng W, Bai Y, Huang L. Selenium nanoparticles fabricated in Undaria pinnatifida polysaccharide solutions induce mitochondria-mediated apoptosis in A375 human melanoma cells. Colloids Surf B Biointerfaces. 2008;67(1):26-31.

24. Huang B, Zhang J, Hou J, Chen C. Free radical scavenging efficiency of nano-Se in vitro. Free Radic Biol Med. 2003;35(7):805-813.

25. Li Q, Chen T, Yang F, Liu J, Zheng W. Facile and controllable one-step fabrication of selenium nanoparticles assisted by L-cysteine. Mater Lett. 2010;64(5):614-617.

26. Zhang Y, Wang J, Zhang L. Creation of highly stable selenium nanoparticles capped with hyperbranched polysaccharide in water. Langmuir. 2010;26(22):17617-17623.

27. Shen Y, Wang X, Xie A, Huang L, Zhu J, Chen L. Synthesis of dextran/ Se nanocomposites for nanomedicine application. Mater Chem Phys. 2008;109(2-3):534-540.

28. Bakshi MS, Kaur H, Banipal TS, Singh N, Kaur G. Biomineralization of gold nanoparticles by lysozyme and cytochrome $\mathrm{C}$ and their applications in protein film formation. Langmuir. 2010;26(16):13535-13544. 
29. Bai Y, Wang Y, Zhou Y, Li W, Zheng W. Modification and modulation of saccharides on elemental selenium nanoparticles in liquid phase. Mater Lett. 2008;62(15):2311-2314.

30. Elhadj S, Chernov AA, De Yoreo JJ. Solvent-mediated repair and patterning of surfaces by AFM. Nanotechnology. 2008;19(10):105304.

31. Lu Q, Gao F, Komarneni S. A green chemical approach to the synthesis of tellurium nanowires. Langmuir. 2005;21(13):6002-6005.

32. Wong HL, Bendayan R, Rauth AM, Xue HY, Babakhanian K, Wu XY. A mechanistic study of enhanced doxorubicin uptake and retention in multidrug resistant breast cancer cells using a polymer-lipid hybrid nanoparticle system. J Pharmacol Exp Ther. 2006;317(3):1372-1381.

33. Min KH, Park K, Kim YS, et al. Hydrophobically modified glycol chitosan nanoparticles-encapsulated camptothecin enhance the drug stability and tumor targeting in cancer therapy. $J$ Control Release. 2008;127(3):208-218.
34. Chen T, Wong YS. Selenocystine induces apoptosis of A375 human melanoma cells by activating ROS-mediated mitochondrial pathway and p53 phosphorylation. Cell Mol Life Sci. 2008;65(17):2763-2775.

35. Chen T, Wong YS. Selenocystine induces caspase-independent apoptosis in MCF-7 human breast carcinoma cells with involvement of p53 phosphorylation and reactive oxygen species generation. Int J Biochem Cell Biol. 2009;41(3):666-676.

36. King KL, Cidlowski JA. Cell cycle regulation and apoptosis. Annu Rev Physiol. 1998;60:601-617.

37. Compton MM. A biochemical hallmark of apoptosis: internucleosomal degradation of the genome. Cancer Metastasis Rev. 1992;11(2): 105-119.

\section{Publish your work in this journal}

The International Journal of Nanomedicine is an international, peerreviewed journal focusing on the application of nanotechnology in diagnostics, therapeutics, and drug delivery systems throughout the biomedical field. This journal is indexed on PubMed Central, MedLine, CAS, SciSearch ${ }^{\circledR}$, Current Contents ${ }^{\circledR} /$ Clinical Medicine,
Journal Citation Reports/Science Edition, EMBase, Scopus and the Elsevier Bibliographic databases. The manuscript management system is completely online and includes a very quick and fair peer-review system, which is all easy to use. Visit http://www.dovepress.com/ testimonials.php to read real quotes from published authors. 